\title{
Evaluation of Anticaries Efficacy of Various Fluoride Varnishes on Artificial Enamel Lesion: An In Vitro Study
}

\author{
Kavya Rani BS ${ }^{1}$, Pavithra K Ramanna ${ }^{2}$, Shilpa Mailankote ${ }^{3}$, Arun K Joy ${ }^{4}$, Archana A Thomas ${ }^{5}$, Mathews Baby ${ }^{6}$
}

\begin{abstract}
Aim: Aim of the current study was to assess the anticariogenic effectiveness of different fluoride varnishes on artificially induced enamel lesions employing scanning electron microscope.

Materials and methods: Eighty healthy, normal premolars without dental caries that were extracted in course of orthodontic therapy with all the surfaces intact were included in this study. A window, $4 \times 4 \mathrm{~mm}$, was made discernible on the buccal surface of each sample tooth. A demineralizing solution at $37^{\circ} \mathrm{C}$ was used to immerse the teeth for 48 hours to induce artificial lesions on the surface of the enamel. Following preparation of the artificial enamel lesions, the 80 premolar teeth were allocated into the four groups (20 each) depending on the fluoride varnish system used as Group I: control, Group II: Duraphat varnish, Group III: MI Varnish, and Group IV: Clinpro White Varnish. The anticariogenic effectiveness of different fluoride varnishes was evaluated employing a scanning electron microscope (SEM).

Results: The MIVarnish (fluoride varnish) group exhibited slightly greater ( $127.20 \pm 0.14)$ mean demineralized lesions, pursued by Clinpro White Varnish use $(126.88 \pm 0.09)$, the control group $(126.36 \pm 0.10)$ and the Duraphat varnish $(124.14 \pm 0.08)$ in that order. Greater mean areas of remineralization were found with use of MI Varnish $(92.40 \pm 0.09)$, pursued by the Duraphat varnish use (106.68 \pm 0.12$)$, use of Clinpro White Varnish $(112.36 \pm 0.08)$, and then the control group $(123.08 \pm 0.18)$ in that order. Statistically significant differences were noted between the experimental groups employing the various fluoride varnishes $(p<0.001)$.

Conclusion: The current research concluded that the MI Varnish group presented a superior protective potential in comparison with Duraphat varnish and Clinpro White Varnish groups.

Clinical significance: Mineral exchanges among teeth and saliva render incipient enamel lesions reversible. A 5\% sodium fluoride varnish is the MI Varnish that is composed of casein phosphopeptide-amorphous calcium phosphate (CPP-ACP) to provide an excellent fluoride varnish that makes available additional bioavailable fluoride, calcium, and phosphate. Therefore, it may be fruitfully utilized in remineralization of initial carious lesions. CPP-ACP may be employed in clinical practice for drawing back or ceasing initial carious lesions.
\end{abstract}

Keywords: Demineralization, Enamel lesion, Fluoride varnish, Scanning electron microscope.

The Journal of Contemporary Dental Practice (2021): 10.5005/jp-journals-10024-3147

\section{INTRODUCTION}

Dental caries is among the frequent bacterial pathosis encountered all over the world. Dental decay has occurred in about 60 to $90 \%$ of children in the school-age group as approximately calculated by the World Health Organization (WHO). Dental decay is characterized by loss of tooth structure owing to demineralization and is multifactorial in nature, occurring in both adults and children. The chief etiologies are the existence of caries causing endogenous bacteria, regular intake of carbohydrates that are fermentable coupled with the presence of a vulnerable host and tooth. ${ }^{1}$

One of the majorly trusted ingredients employed in preventing tooth decay is fluoride that acts by hardening the mineralized tooth surface, thereby preventing its succession. The utility of fluoride as a vehicle for topical use is prolonging the time of contact with the tooth enamel. ${ }^{2}$ The quantity of fluoride thus maintained within the enamel structure is higher, thereby decreasing demineralization and promoting remineralization. Some evidence also implicates that fluoride may have an effect on plaque-microbial flora metabolism and their retention onto the tooth surface, prohibition of the bacterial enzymes, decreased bacterial growth, and perhaps a bactericidal action. ${ }^{3}$

In recent times, a large variety of topical fluoride systems for proficient applications are available and include gels, rinses, drops, varnishes, and foams. The fluoride varnishes characteristically

\footnotetext{
${ }^{1}$ Department of Pedodontics and Preventive Dentistry, College of Dental Sciences, Davanagere, Karnataka, India

${ }^{2}$ Department of Prosthodontics, Crown and Bridge and Implantology, Vydehi Institute of Dental Sciences and Research Hospital, Bengaluru, Karnataka, India

${ }^{3}$ Department of Public Health Dentistry, AB Shetty Memorial Institute of Dental Sciences (ABSMIDS), NITTE (Deemed to be University), Mangaluru, Karnataka, India

${ }^{4}$ Department of Prosthodontics, St Gregorios Dental College, Chelad, Kothamangalam, Kerala, India

${ }^{5}$ Department of Pediatric and Preventive Dentistry, KMCT Dental College, Calicut, Kerala, India

${ }^{6}$ Department of Public Health Dentistry, Mar Baselios Dental College, Kothamangalam, Kerala, India
}

Corresponding Author: Kavya Rani BS, Department of Pedodontics and Preventive Dentistry, College of Dental Sciences, Davanagere, Karnataka, India, Phone: +91 9901647816, e-mail:dr.kavyarani@gmail.com

How to cite this article: Rani KBS, Ramanna PK, Mailankote S, et al. Evaluation of Anticaries Efficacy of Various Fluoride Varnishes on Artificial Enamel Lesion: An In Vitro Study. J Contemp Dent Pract 2021;22(7):774-777.

Source of support: Nil

Conflict of interest: None

(c) Jaypee Brothers Medical Publishers. 2021 Open Access This article is distributed under the terms of the Creative Commons Attribution 4.0 International License (https://creativecommons.org/licenses/by-nc/4.0/), which permits unrestricted use, distribution, and non-commercial reproduction in any medium, provided you give appropriate credit to the original author(s) and the source, provide a link to the Creative Commons license, and indicate if changes were made. The Creative Commons Public Domain Dedication waiver (http://creativecommons.org/publicdomain/zero/1.0/) applies to the data made available in this article, unless otherwise stated. 
comprise $5 \%$ sodium fluoride and provide a comparatively simple vehicle with the ability to deliver cariostatic quantity of fluoride. A varnish that contains fluoride presents numerous benefits as compared to other techniques used to apply fluoride on susceptible teeth. These advantages include efficacy, safety, and simplicity of applications. Additionally, extended time of contact with the tooth surfaces coupled with long-lasting fluoride discharge renders the use of fluoride varnishes superior to other techniques of fluoride application. $^{4}$

Fluoride varnishes fortified with extra calcium as well as phosphate components have been lately launched with an aim of enhancing calcium and phosphate quantity within saliva, thereby improving the ability to prevent demineralization and promote probable remineralization of the lesion. ${ }^{5}$ Giving consideration to such modifications in the composition of sodium fluoride varnishes, the effects of adding calcium and phosphate in enhancing or deteriorating the effects of fluoride remain ambiguous. Hence, this research was performed to evaluate the anticariogenic effectiveness of different fluoride varnishes on artificially induced enamel lesions.

\section{Materials and Methods}

\section{Sample Selection}

The current in vitro research was performed in the Department of Pedodontics and Preventive Dentistry, Sri Siddhartha Dental College and Hospital, Karnataka, India. Eighty healthy, normal premolars without dental caries that were extracted in course of orthodontic therapy with all the surfaces intact were included in this study. The exclusion criteria included noticeable cracks on the tooth surface, presence of hypoplastic regions, white spot lesions, and dental caries. The teeth were subjected to a meticulous ultrasonic scaling followed by polishing with the use of a rubber cup and oral prophylaxis paste. Sterilization was performed, after which the sample teeth were placed in $1 \%$ thymol solution until further preparation.

\section{Enamel Window Preparation}

After all the samples were subjected to polishing, a 1-mm cut was made $1 \mathrm{~mm}$ beneath the cementoenamel junction (CEJ) on the buccal surfaces. The samples were then embedded in acrylic blocks such that the coronal portions were seen. A window, $4 \times 4 \mathrm{~mm}$, was made discernible on the buccal surface of each sample tooth and subjected to glazing with nail varnish except the window portion that was later on assessed for differences in values following demineralization/remineralization.

\section{Preparation of Artificial Demineralized Area on Enamel Surface}

A solution containing $50 \mathrm{mM}$ acetic acid ( $\mathrm{pH}$ 4.5), $2.2 \mathrm{mM}$ potassium dihydrogen phosphate $\left(\mathrm{KH}_{2} \mathrm{PO}_{4}\right), 2.2 \mathrm{mM}$ calcium nitrate $\left(\mathrm{Ca}\left(\mathrm{NO}_{3}\right)_{2}\right)$, and $0.1 \mathrm{ppm} \mathrm{NaF}$ at $37^{\circ} \mathrm{C}$ was used to immerse the teeth for 48 hours to induce artificial lesions on the surface of the enamel. ${ }^{6}$ The $\mathrm{pH}$ of this solution was adjusted to 5.0 with the use of potassium hydroxide. Each sample was subjected to rinsing using deionized water for about 60 seconds after demineralization. The teeth were dried employing a tissue and preserved at about $100 \%$ relative humidity and $4^{\circ} \mathrm{C}$ until called for use.

\section{Application of Fluoride Varnish}

Following preparation of the artificial enamel lesions, 80 premolar teeth were allocated into the four groups (20 each) depending on the fluoride varnish system used and mean demineralized areas for each group were calculated before the application of fluoride varnish using a scanning electron microscope (SEM) at $1000 \times$ magnification.

- Group I: Control

- Group II: Duraphat ${ }^{\circledR}$ varnish (Colgate-Palmolive, New York, USA)

- Group III: MI Varnish ${ }^{\mathrm{TM}}$ (GC Corporation, Tokyo, Japan)

- Group IV: Clinpro White Varnish (3M ESPE, St Paul, Minnesota, USA).

Each sample of every experimental group was subjected to treatment with the concerned fluoride varnish with the exception of the control group for 7 days, every 24 hours using a cotton application tip. Every day, the individual sample was chafed with the concerned varnish for 4 minutes, followed by the removal of extra varnish with gentle air pressure, washing under deionized water, and placement in artificial saliva. The artificial saliva was composed of $1.45 \mathrm{mM} \mathrm{CaCl} 2 \cdot 2 \mathrm{H}_{2} \mathrm{O}, 5.40 \mathrm{mM} \mathrm{KH}_{2} \mathrm{PO}_{4}, 14.90 \mathrm{mM} \mathrm{KCl}, 28.40 \mathrm{mM}$ $\mathrm{NaCl}$, and $2.20 \mathrm{~g} / \mathrm{L}$ mucin; that was set at $\mathrm{pH} 6.8$ by adding $5 \mathrm{M}$ sodium hydroxide. ${ }^{7}$ For the control group, sample teeth were only subjected to washing with deionized water followed by placement in artificial saliva. Artificial saliva was subjected to change once every 24 hours prior to immersion of newly treated specimens.

\section{Evaluation of an Anticaries Activity of Fluoride Varnishes Using Scanning Electron Microscope}

Seven days later, the samples of every group were subjected to SEM examination. Observation under SEM was done by trained, experienced, and standardized observers who were blinded with regard to the experimental groups. The $100 \%$ ethanol solution was employed for specimen dehydration and was then placed in a furnace at $60^{\circ} \mathrm{C}$ for 24 hours. Following this, the specimen teeth were subjected to sputter coating with gold in vacuum metalizing equipment. Examination was done using a SEM at $1000 \times$ magnification and assessed on the basis of the below-mentioned criteria.

- Score 0-Enamel surface was ideally intact without grooves, pits, and porosity.

- Score 1-Enamel surface exhibited irregularities, with no demineralization of prismatic and/or interprismatic enamel.

- Score 2-Presence of wrinkles and demineralization of prismatic/interprismatic enamel.

- Score 3-Diffuse demineralization involving the rod core, with breakdown of prism morphologic characteristics.

\section{Statistical Analysis}

The data were analyzed statistically using the SPSS Statistics for Windows Software, version 20. Descriptive statistics was used to calculate the mean and standard deviation. Analysis of variance (ANOVA) and Tukey's post hoc test were employed in the assessment of the comparative efficacy of the different fluoride varnishes. The significance point was set at $5 \%$.

\section{Results}

Table 1 shows the assessment of mean demineralized areas among the different fluoride varnish study groups. The MI Varnish group exhibited slightly greater (127.20 \pm 0.14$)$ mean demineralized lesions, pursued by Clinpro White Varnish use (126.88 \pm 0.09$)$, the control group $(126.36 \pm 0.10)$ and the Duraphat varnish $(124.14 \pm 0.08)$ in that order. No statistically significant differences were noted between the experimental groups. 
Table 1: Evaluation of mean demineralized lesion among various fluoride varnish groups

\begin{tabular}{llll}
\hline Fluoride varnish groups & Mean $\pm S D$ & Fvalue & pvalue \\
\hline Group I: Control & $126.36 \pm 0.10$ & & \\
Group II: Duraphat varnish & $124.14 \pm 0.08$ & & \\
Group III: MI Varnish & $127.20 \pm 0.14$ & & 0.729 \\
Group IV: Clinpro White Varnish & $126.88 \pm 0.09$ & & \\
\hline
\end{tabular}

Table 2: Evaluation of mean remineralized areas among various fluoride varnish groups after 7 days

\begin{tabular}{lrrr}
\hline Fluoride varnish groups & \multicolumn{1}{c}{ Mean $\pm S D$} & Fvalue & pvalue \\
\hline Group I: Control & $123.08 \pm 0.18$ & & \\
Group II: Duraphat varnish & $106.68 \pm 0.12$ & & \\
Group III: MI Varnish & $92.40 \pm 0.09$ & 24.912 & 0.001 \\
Group IV: Clinpro White Varnish & $112.36 \pm 0.08$ & & \\
\hline
\end{tabular}

Table 3: Comparison of fluoride varnish efficacy using Tukey's HSD test

\begin{tabular}{llcl}
\hline Groups & Compared with & Mean difference (I-J) & Sig. \\
\hline Control & Duraphat varnish & 16.40 & 0.001 \\
& MI Varnish & 30.68 & 0.001 \\
& Clinpro White & 10.72 & 0.001 \\
& Varnish & & \\
Duraphat & Control & -16.40 & 0.001 \\
varnish & MI Varnish & 14.28 & 0.001 \\
& Clinpro White & -5.68 & 0.084 \\
& Varnish & & \\
MIVarnish & Control & -30.68 & 0.001 \\
& Duraphat varnish & -14.28 & 0.001 \\
& Clinpro White & -19.96 & 0.001 \\
& Varnish & & \\
Clinpro White & Control & -10.72 & 0.001 \\
Varnish & Duraphat varnish & 5.68 & 0.084 \\
& MI Varnish & 19.96 & 0.001 \\
\hline
\end{tabular}

Table 2 demonstrates the mean areas of remineralization amid the different fluoride varnish experimental groups on evaluation following 7 days. Greater mean areas of remineralization were found with the use of MI Varnish $(92.40 \pm 0.09)$, pursued by the Duraphat varnish use $(106.68 \pm 0.12)$, the use of white varnish (112.36 \pm 0.08$)$, and then the control group $(123.08 \pm 0.18)$, respectively. Statistically significant differences were noted between the experimental groups employing the various fluoride varnishes ( $p<0.001)$.

Table 3 displays the global comparative assessment of the effectiveness of the fluoride varnishes employing Tukey's honestly significant difference (HSD) test. With the exception of Duraphat varnish and the Clinpro White Varnish, statistically significant differences ( $p<0.001$ ) were noted between the study groups.

The inference of the present study indicates that the MI Varnish group offered a greater anticariogenic potential compared to Duraphat varnish group and Clinpro White Varnish group.

\section{Discussion}

Faster diagnosis of early carious lesions has given birth to a novel era in preventive dental practice. Since the 1930s, the efficiency of fluoride in preventing dental caries is well established. In recent years, topical application of fluoride agents has had an upper edge in the prevention of caries progression. Varnishes composed of fluoride were established in the late 1960s and beginning of the 1970s to improvise the inadequacies of the prevailing topical fluoride agents, including fluoride-containing mouth rinses and gels, by lengthening the time of contact between enamel and fluoride. A chief characteristic of varnish is that owing to its resin base that suspends the fluoride, it is persistent in adhering to the teeth, thereby permitting lengthy interaction between fluoride and enamel over time. ${ }^{8}$

It is a well-known fact that fluoride can augment remineralization rate of teeth and cause the redeposition of calcium as well as phosphate ions on the surface of the enamel. A protection to enamel is provided by fluoride adhering to the tooth surface that has not been demineralized against attack from acid-producing bacteria, avoiding demineralization, and boosting the process of remineralization. This happens from the attraction of phosphate and calcium ions, thereby provoking mineral development. ${ }^{9}$

Concentrated fluoride varnishes have a dissimilar mechanism of action. Ogaard et al. ${ }^{10}$ state that systems that have greater fluoride content on application cause the production of an intermediary, namely calcium fluoride over the surface of teeth, within plaques, and on enamel that is demineralized. Beneath a SEM, the calcium fluoride structures thus produced can be noticed as tiny globules over the enamel surface after applying the varnish.

In the current research, greater mean areas of remineralization were found following the application of MI Varnish, pursued by Duraphat varnish, Clinpro White Varnish, and the control group in that order. The active component of MI Varnish is NaF and Casein phosphopeptide-amorphous calcium phosphate (CPP-ACP). CPP-ACP is a well-documented reservoir of phosphate as well as calcium in close proximity to the areas of probable demineralization and prohibits demineralization or enhances remineralization or performs both according to Patil et al. ${ }^{11}$ A likely action to avoid demineralization is that casein has the ability to buffer plaque acid through direct or indirect bacterial catabolism. Casein can yield amino acids that take protons, thereby acting as buffers.

Schemehorn et al. ${ }^{12}$ and Hicks et al. ${ }^{13}$ have cited that MI Varnish is superior to Clinpro White Varnish and may tender a higher anticariogenic capability. This is attributed to the integration of nanocomplexes within plaque and enamel surfaces by CPP-ACP. Also, it has been documented by Pithon et al. ${ }^{14}$ that MI Varnish ( $5 \%$ sodium fluoride varnish fortified with CPP-ACP) is more efficient when compared to Duraphat ( $5 \%$ sodium fluoride varnish without (PP-ACP) in decreasing the depth of dental caries. A research assessing the fluoride, calcium, and inorganic phosphate ion release from fluoride varnishes having dissimilar composition (MI Varnish, Clinpro White Varnish, Enamel Pro Varnish, Duraphat, and Bifluorid 5), by Cochrane et al., ${ }^{15}$ concluded that calcium/ fluoride ion was greatest with the MI Varnish.

Shen et al. ${ }^{16}$ assessed the capability of calcium phosphate and fluoride consisting varnishes in the prevention of enamel demineralization. MI Varnish with its fluoride and CPP-ACP content was better than other varnishes in providing protection from enamel demineralization that is in agreement with our findings.

In the current study, remineralizing capability was found to be superior with the use of Duraphat varnish vs the Clinpro White Varnish. Research by Attin et al. ${ }^{17}$ and Delbem et al. ${ }^{18}$ have shown that formed calcium fluoride was lesser than retained calcium fluoride in these groups. $\mathrm{pH}$ cycling prototype presents a highacid trial and was hence incorporated in this study. The more 
the demineralization of enamel, the higher is its reactivity, which promotes fluoride deposition from the solution although the fluoride content is low.

The limitation of the present study is an in vitro research in which the clinical as well as physiological processes cannot be fully replicated. The uptake of fluoride by enamel was not studied. The subtleties of human saliva as influencing the fluoride release rate in the in vitro setup were not given consideration. Hence, future research bearing in mind these oral factors, antibacterial capacity, and safety in a clinical setup is suggested.

\section{Conclusion}

Amid the limitations of this study, the current research concluded that MI Varnish group presented a superior protective potential in comparison with Duraphat varnish and Clinpro White Varnish group.

\section{References}

1. Baik A, Alamoudi N, El-Housseiny A, et al. Fluoride varnishes for preventing occlusal dental caries: a review. Dent J (Basel) 2021;9(6):64. DOI: 10.3390/dj9060064.

2. Gao SS, Zhang S, Mei ML, et al. Caries remineralisation and arresting effect in children by professionally applied fluoride treatment: a systematic review. BMC Oral Health 2016;16(12):1-9. DOI: 10.1186/ s12903-016-0171-6.

3. Hazelrigg CO, Dean JA, Fontana M. Fluoride varnish concentration gradient and its effect on enamel demineralization. Pediatr Dent 2003;25(2):119-126. https://pubmed.ncbi.nlm.nih.gov/12723836/

4. Lippert F, Hara AT, Martinez-Mier EA, et al. Laboratory investigations into the potential anticaries efficacy of fluoride varnishes. Pediatr Dent 2014;36(4):291-295. https://pubmed.ncbi.nlm.nih.gov/25197993/

5. Larsen MJ, Pearce EIF. Saturation of human saliva with respect to calcium salts. Arch Oral Biolog 2003;48(4):317-322. DOI: 10.1016/ s0003-9969(03)00007-4.

6. Nozari A, Ajami S, Rafiei A, et al. Impact of nano hydroxyapatite, nano silver fluoride and sodium fluoride varnish on primary teeth enamel remineralization: an in vitro study. J Clin Diagn Res 2017;11(9):ZC97ZC100. DOI: 10.7860/JCDR/2017/30108.10694.

7. Wong $\mathrm{L}$, Sissions $\mathrm{C}$. A comparison of human dental plaque microcosm biofilms grown in an undefined medium and a chemically defined artificial saliva. Arch Oral Biol 2001;46(6):477-486. DOI: 10.1016/s00039969(01)00016-4.

8. Miller EK, Vann WF Jr. The use of fluoride varnish in children: A critical review with treatment recommendations. J Clin Pediatr Dent 2008;32(4):259-264. DOI: 10.17796/jcpd.32.4.a7516vj8762687v5.

9. Jeevarathan J, Deepti A, Muthu MS, et al. Effect of fluoride varnish on Streptococcus mutans counts in plaque of caries-free children using Dentocult SM strip mutans test: a randomized controlled triple blind study. J Indian Soc Pedod Prev Dent 2007;25(4):157-163. DOI: 10.4103/0970-4388.37010.

10. Ogaard B, Rolla G, Helgeland K. Fluoride retention in sound and demineralized enamel in vivo after treatment with a fluoride varnish (Duraphat). Scand J Dent Res 1984;92(3):190-197. DOI: 10.1111/j.16000722.1984.tb00878.x.

11. Patil N, Choudhari S, Kulkarni S, et al. Comparative evaluation of remineralizing potential of three agents on artificially demineralized human enamel: an in vitro study. J Conserv Dent 2013;16(2):116-120. DOI: 10.4103/0972-0707.108185.

12. Schemehorn BR, Wood GD, McHale W, et al. Comparison of fluoride uptake into tooth enamel from two fluoride varnishes containing different calcium phosphate sources. J Clin Dent 2011;22(2):51-54. https://pubmed.ncbi.nlm.nih.gov/21702326/

13. Hicks J, Garcia-Godoy F, Flaitz C. Biological factors in dental caries: role of remineralization and fluoride in the dynamic process of demineralization and remineralization (part 3). J Clin Pediatr Dent 2004;28(3):203-214. DOI: 10.17796/jcpd.28.3.w06104271746j34n.

14. Pithon MM, Dos Santos MJ, Andrade CS, et al. Effectiveness of varnish with CPP-ACP in prevention of caries lesions around orthodontic brackets: an OCT evaluation. Eur J Orthod 2015;37(2):177-182. DOI: 10.1093/ejo/cju031.

15. Cochrane NJ, Shen $P$, Yuan $Y$, et al. Ion release from calcium and fluoride containing dental varnishes. Aust Dent J 2014;59(1):100-105. DOI: 10.1111/adj.12144.

16. Shen $P$, Bagheri $R$, Walker $G D$, et al. Effect of calcium phosphate addition to fluoride containing dental varnishes on enamel demineralization. Aust Dent J 2016;61(3):357-365. DOI: 10.1111/adj.12385.

17. Attin $\mathrm{T}$, Hartmann $\mathrm{O}$, Hilgers $\mathrm{RD}$, et al. Fluoride retention of incipient enamel lesions after treatment with a calcium fluoride varnish in vivo. Arch Oral Biol 1995;40(3):169-174. DOI: 10.1016/00039969(95)98804-8.

18. Delbem AC, Brighenti FL, Oliveira FA, et al. In vitro assessment of an experimental coat applied over fluoride varnishes. J Appl Oral Sci 2009;17(4):280-283. DOI: 10.1590/s1678-77572009000400003. 